Case

Report

\title{
An Intrathoracic Low-Grade Fibromyxoid Sarcoma Arising from the Chest Wall with Massive Pleural Effusion
}

\author{
Yoshiaki Tominaga, MD, ${ }^{1}$ Takashi Eguchi, MD, ${ }^{1}$ Takayuki Shiina, MD, ${ }^{1}$ \\ Kazutoshi Hamanaka, MD, PhD, ${ }^{1}$ Jun Amano, MD, PhD,${ }^{2}$ Shiho Asaka, MD, ${ }^{3}$ \\ and Kazuo Yoshida, $\mathrm{MD}, \mathrm{PhD}^{1}$
}

\begin{abstract}
We herein report a case of an intrathoracic low-grade fibromyxoid sarcoma arising from the chest wall and associated with massive pleural effusion. A 70-year-old man presented with a persistent cough. A chest computed tomography scan revealed a large mass in the right pleural cavity with massive pleural effusion. No malignant cells were recognized in the pleural effusion by thoracentesis. A malignant soft tissue tumor was suspected, and surgery was performed. The tumor arose from the posterior chest wall and was resected with the connected chest wall. The definitive diagnosis was a low grade fibromyxoid sarcoma. Because the posterior margin of the chest wall was microscopically tumor positive, postoperative irradiation was performed. The patient has now been followed up for 30 months with no evidence of recurrence.
\end{abstract}

Keywords: low-grade fibromyxoid sarcoma, intrathoracic tumor, chest wall resection, pleural effusion

\section{Introduction}

Low-grade fibromyxoid sarcoma (LGFMS) is a relatively rare tumor, and few cases have been reported previously. We herein report a case of an intrathoracic LGFMS arising from the chest wall associated with a massive pleural effusion.

${ }^{1}$ Department of Thoracic Surgery, Shinshu University School of Medicine, Nagano, Japan

${ }^{2}$ Department of Surgery, Shinshu University School of Medicine, Nagano, Japan

${ }^{3}$ Department of Pathology, Shinshu University School of Medicine, Nagano, Japan

Received: October 31, 2012; Accepted: January 16, 2013 Corresponding author: Takashi Eguchi, MD. Department of Thoracic Surgery, Shinshu University School of Medicine, Asahi 3-1-1, Matsumoto, Nagano 390-8621, Japan

Email: tks1976@shinshu-u.ac.jp

(C)2014 The Editorial Committee of Annals of Thoracic and Cardiovascular Surgery. All rights reserved.

\section{Case Report}

A 70-year-old man presented at our affiliated hospital with a persistent cough. He had a medical history of prostate cancer. A chest radiograph revealed a massive pleural effusion of the right hemithorax (Fig. 1a). A chest computed tomography scan revealed a large mass, $17 \mathrm{~cm}$ in size, in the right pleural cavity with a massive pleural effusion (Fig. 1b). By magnetic resonance imaging, the tumor showed a heterogeneous appearance with a low to slightly high signal intensity on a T1-weighted image, and an intermediate to high signal intensity on a T2-weighted image (Fig. 2). By ${ }^{18}$ F-fluorodeoxyglucose positron emission tomography, a high level of ${ }^{18} \mathrm{~F}$-fluorodeoxyglucose accumulation was recognized, and the maximum standardized uptake value was measured at 12.4.

No malignant cells were identifiable in the pleural effusion by thoracentesis. In the examination of the pleural effusion, the protein level was $4.4 \mathrm{~g} / \mathrm{dl}$, the lactate dehydrogenase level was $241 \mathrm{IU} / \mathrm{L}$, the amylase level 


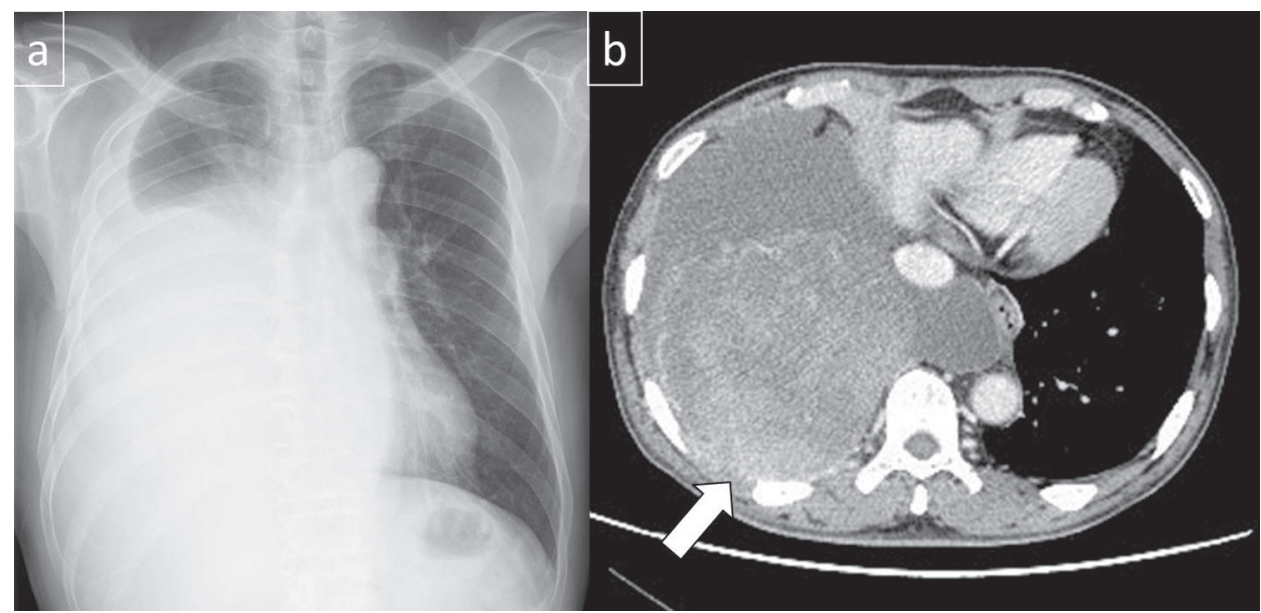

Fig. 1 (a) Chest radiograph revealing a massive pleural effusion of the right lung. (b) Chest computed tomography showing a large mass (arrow) in the right pleural cavity with a massive pleural effusion.

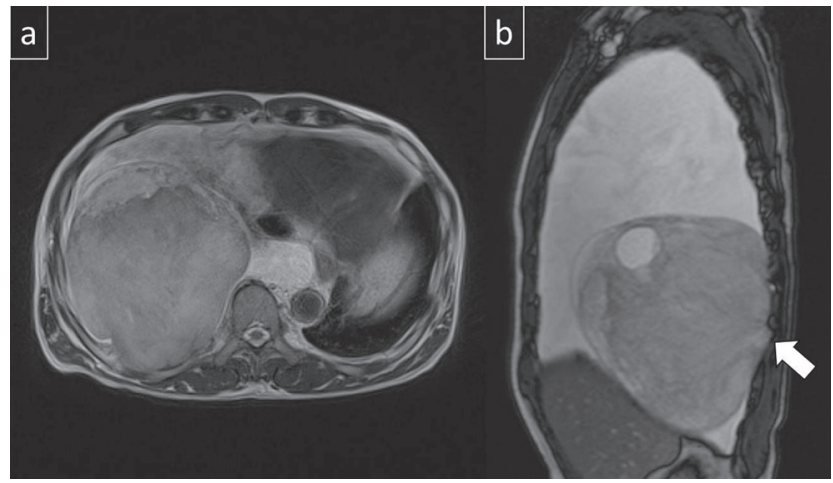

Fig. 2 (a) Magnetic resonance imaging showing the tumor presenting with a heterogeneous appearance with an intermediate to high signal intensity on T2-weighted images. (b) Sagittal section of the tumor revealing a bulging outward into the intercostal space of the posterior chest wall (arrow).

was $48 \mathrm{U} / \mathrm{L}$, the cholesterol level was $83 \mathrm{mg} / \mathrm{dl}$, the triglyceride level was $26 \mathrm{mg} / \mathrm{dl}$, the glucose level was $206 \mathrm{mg} / \mathrm{dl}$, and the $\mathrm{pH}$ was 8.0. The pleural:serum lactate dehydrogenase ratio was 1.43 , and the pleural:serum protein ratio was 0.62 . A malignant soft tissue tumor was suspected, and surgery was performed. Initially, a large amount $(2000 \mathrm{ml})$ of the pleural effusion was drained. The tumor arose from the posterior chest wall and was, therefore, resected with the connected chest wall. Three ribs, from the ninth to eleventh, were resected, and the chest wall defect was covered with polytetrafluoroethylene (Gore-Tex) mesh. A surgical margin greater than $4 \mathrm{~cm}$ was secured, but a $4 \mathrm{~cm}$ distance could not be achieved on the vertebral side in order to preserve the vertebral bodies. The lengths of the resected ninth, tenth,

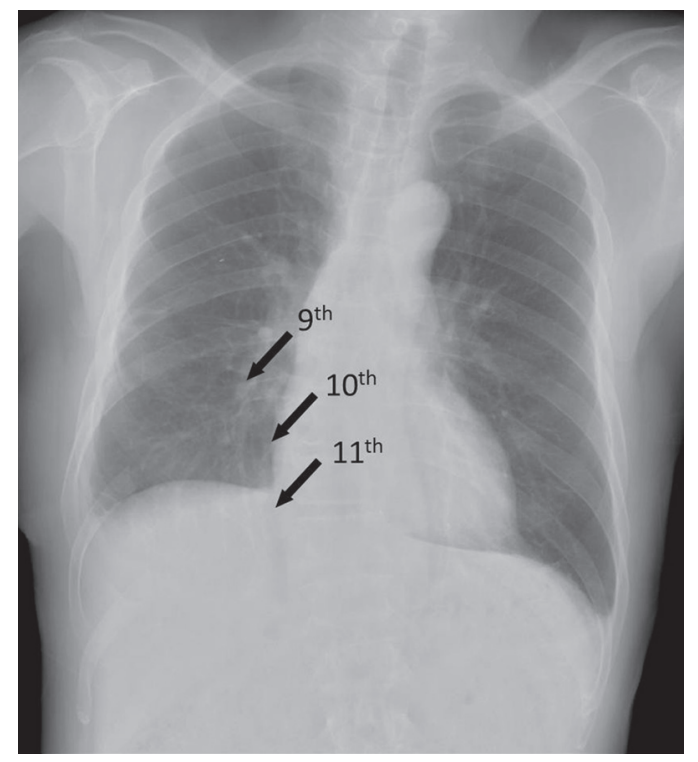

Fig. 3 A postoperative radiograph of the patient showing defects of the ninth to eleventh ribs. The posterior margins of these ribs are indicated by arrows.

and eleventh ribs were approximately $8 \mathrm{~cm}, 10 \mathrm{~cm}$, and $10 \mathrm{~cm}$, respectively, and the closest distance between the posterior margins of the vertebral bodies was less than $1 \mathrm{~cm}$ (Fig. 3). Subsequent pathological examinations revealed an $18 \times 15 \times 10 \mathrm{~cm}$ tumor consisting of proliferated spindle cells with an admixture of heavily collagenized, hypocellular areas and more cellular myxoid areas (Fig. 4). Immunohistochemically, the tumor did not express S100 protein, cytokeratins, or CD34.

The definitive diagnosis in this case was a low grade fibromyxoid sarcoma. Because the proliferation of a few 


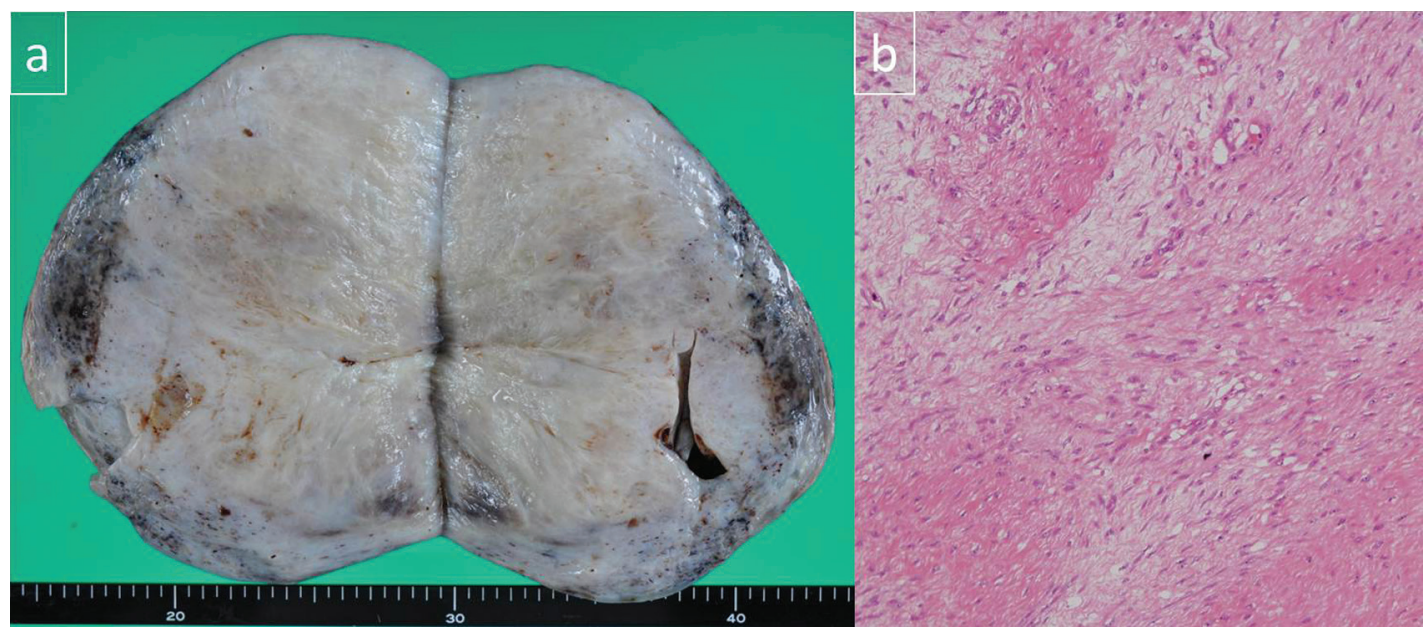

Fig. 4 (a) Divided surface of the tumor showing an ash gray solid mass with partial hemorrhaging and cystic lesions. (b) Histological examination (hematoxylin and eosin staining; original magnification, $20 \times$ ) revealing proliferated spindle cells with an admixture of heavily collagenized areas and myxoid areas.

spindle cells was recognized microscopically in the periosteum around the posterior margin of the chest wall, postoperative irradiation (60 Gy) was done. The patient was subsequently followed up for 30 months with no evidence of recurrence.

\section{Discussion}

LGFMS is a relatively rare soft tissue tumor that was first described by Evans in 1987.1) This lesion typically occurs in the proximal extremities or trunk ${ }^{2)}$ but is rarely described as a primary neoplasm within the thoracic cavity. Indeed, only two cases of intrathoracic LGFMS arising from the chest wall have been reported previously. ${ }^{3,4)}$

Steiner, et al. have reported a case of a 12-year-old girl who developed a huge LGFMS that occupied the entire hemithorax with acute respiratory distress. ${ }^{4}$ Following surgery in that case, the mass was revealed to be attached to the posterior lateral chest wall, but a combined chest wall resection was not performed and the tumor margin was positive. In contrast, Higuchi, et al. reported a case of an intrathoracic LGFMS which was successfully treated using a combined chest wall resection. ${ }^{3)}$ In our current case, the intrathoracic tumor was attached to the posterior chest wall, and a combined chest wall resection was performed. However, on the vertebral side, the margin was microscopically tumor-positive, likely because of an insufficient distance from the tumor.

Generally, $4 \mathrm{~cm}$ to $5 \mathrm{~cm}$ from the macroscopic tumor border is recommended for a lateral excision of a chest wall tumor to achieve a microscopic negative margin.
This is because malignant cells can spread within the marrow cavity, or along the periosteum or parietal pleura. ${ }^{5)}$ However, as observed in our current case, if a negative margin resection cannot be achieved without sacrificing structures that are vital to anatomic function or cosmesis, the tumor is resected away from critical structures with microscopically positive margins, and irradiation is employed in these patients. ${ }^{6)}$ In our current case, no evidence of recurrence was evident for 30 months after postoperative irradiation. However, it has been reported that in patients treated using postoperative radiation therapy for soft tissue sarcomas, a tumor located at a site other than the extremity and a lesion greater than $5 \mathrm{~cm}$ in size are suggestive of a poor prognosis. ${ }^{6}$ Moreover, LGFMS lesions may metastasize many years after their initial diagnosis ${ }^{2)}$ and careful and long-term follow up regimens are thus recommended for affected patients.

In our current LGFMS case, a massive pleural effusion was found to be associated with the tumor. To our knowledge, no previous cases of intrathoracic LGFMS with massive pleural effusion have been reported. In our current case, the pleural effusion was exudative, but there was no pleural dissemination and no malignant findings within this effusion. An extensive lung atelectasis possibly caused by tumor compression was also recognized in our current case. It is possible that a lung atelectasis could induce a pleural effusion, but such an atelectasis-induced effusion is reported to be generally transudative. ${ }^{7)}$ The mechanism of accumulation of the massive pleural effusion in our current case remains unclear. 


\section{Conclusions}

Although rare, LGFMS should be considered as a possibility during the differential diagnosis of an intrathoracic tumor. Postoperative irradiation should be considered as a treatment option in cases of LGFMS arising from the chest wall in which a microscopic negative margin cannot be achieved through a combined chest wall resection.

\section{Disclosure Statement}

None to declare.

\section{References}

1) Evans HL. Low-grade fibromyxoid sarcoma. A report of two metastasizing neoplasms having a deceptively benign appearance. Am J Clin Pathol 1987; 88: 615-9.

2) Folpe AL, van den Berg E, Molenaar WM. Low grade fibromyxoid sarcoma. World health organization classification of tumours. Pathology and genetics of tumours of soft tissue and bone. Lyon: IARC Press, 2002; pp 104-5.

3) Higuchi M, Suzuki H, Shio Y, et al. Successfully resected intrathoracic low-grade fibromyxoid sarcoma. Gen Thorac Cardiovasc Surg 2010; 58: 348-51.

4) Steiner MA, Giles HW, Daley WP. Massive low-grade fibromyxoid sarcoma presenting as acute respiratory distress in a 12-year-old girl. Pediatr Radiol 2009; 39: 396-9.

5) Incarbone M, Pastorino U. Surgical treatment of chest wall tumors. World J Surg 2001; 25: 218-30.

6) Delaney TF, Kepka L, Goldberg SI, et al. Radiation therapy for control of soft-tissue sarcomas resected with positive margins. Int J Radiat Oncol Biol Phys 2007; 67: 1460-9.

7) Bartter T, Santarelli R, Akers SM, et al. The evaluation of pleural effusion. Chest 1994; 106: 1209-14. 